\title{
EXPERIMENTAL DETERMINATION OF DUAL-WAVELENGTH MIE LIDAR GEOMETRIC FORM FACTOR COMBINING SIDE-SCATTER AND BACK-SCATTER SIGNALS
}

\author{
Zhenzhu Wang*, Zongming Tao, Dong Liu, Chenbo Xie, and Yingjian Wang
}

Key laboratory of Atmospheric Composition and Optical Radiation, Anhui Institute of Optics and Fine Mechanics, Chinese Academy of Sciences, Hefei, Anhui, 230031 China, *Email: zzwang@aiofm.ac.cn

\begin{abstract}
In theory, lidar overlap factor can be derived from the difference between the particle backscatter coefficient retrieved from lidar elastic signal without overlap correction and the actual particle backscatter coefficient, which can be obtained by other measured techniques. The side-scatter signal using a CCD camera is testified to be a powerful tool to detect the particle backscatter coefficient in near ground layer during night time. In experiment, by combining side-scatter and backscatter signals the geometric form factor for vertically-pointing Mie lidar in $532 \mathrm{~nm}$ channel is determined successfully, which is corrected by an iteration algorithm combining the retrieved particle backscatter coefficient using CCD sidescatter method and Fernald method. In this study, the method will be expanded to $1064 \mathrm{~nm}$ channel in dual-wavelength Mie lidar during routine campaigns. The experimental results in different atmosphere conditions demonstrated that the method present in this study is available in practice.
\end{abstract}

\section{INTRODUCTION}

The incomplete overlap between laser beam and receiver field of view significantly leads to lidar signal loss in the near-field range, and affects the lidar measurement in this range. However, lidar measurement in the near-field range (e.g. particles in the boundary layer) is very essential. The fraction of the laser beam intersecting the receiver field of view is described by geometric form factor (named GFF), and the influence of the incomplete lidar signal can be corrected by GFF. Theoretical and experimental methods have been explored to determine GFF [1-6]. In theoretical methods, GFF is determined by application of a ray-tracing model, and some parameters of the lidar must be known with a sufficient accuracy [1-2], which is not the best idea in practice. In experimental methods, a trustworthy determination of GFF is possible under homogenous atmosphere [3], and this method only can be used when the instrument is oriented horizontally. However, some lidars are only oriented vertically because of complex structure or mobile carrier, and the atmosphere along the laser path is generally inhomogeneous. In order to solve this problem, Ulla Wandinger [4] developed a method based on combined Raman-Mie lidar, and $\mathrm{Hu}$ [5] proposed a method based on nitrogen Raman signal and nitrogen molecule density profile along the laser path. For these two methods, some inherent hypotheses limit their application, as well as some lidars with only Mie channels. Wang [6] introduced an experiment method to determine GFF for vertically-pointing lidar by iterative algorithm based on the CCD side-scatter technique. In this paper, the method will be expanded to $1064 \mathrm{~nm}$ channel in dual-wavelength Mie lidar combining side-scatter and back-scatter signals. The Mie lidar system consists of laser, telescope, PMT, APD, CCD camera, ICC and related optical and mechanical parts as shown in figure 1 , which will not be introduced in detail here.

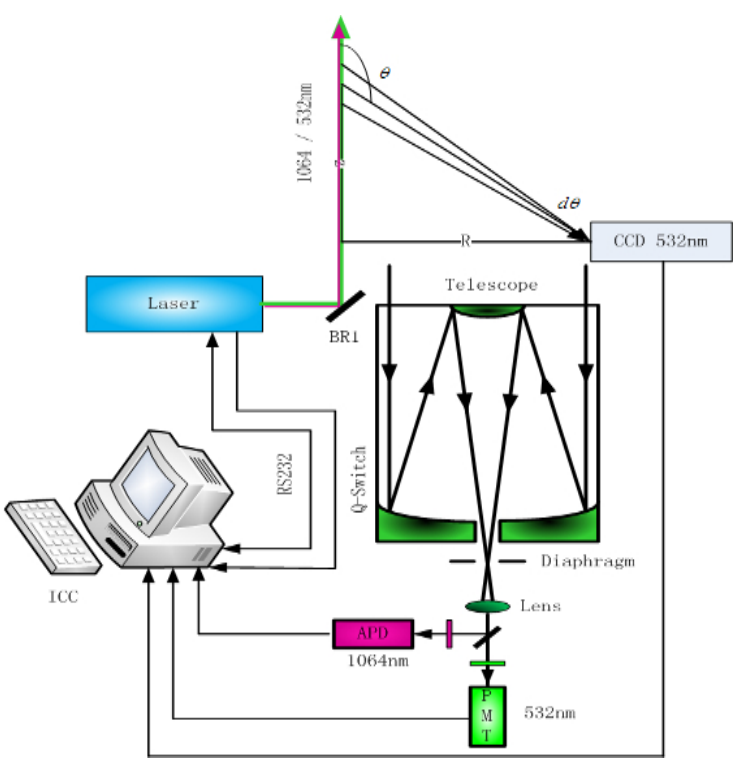

Figure 1. The diagram of lidar system

\section{METHODOLOGY}

The CCD side-scatter technique is a developing technique in which the CCD camera is spatially 
separated to look the laser beam at a certain distance. The side-scatter lidar has no overlap problem as in a backscatter lidar, and is especially suitable to detect actual particle backscatter coefficient in the near range during nighttime [7]. Particle backscatter coefficient can be retrieved from elastic signal using Fernald method [8]. The backscattering coefficient can also be retrieved from side-scatter technique by numerical inversion method [9]. An iterative algorithm is developed to determine GFF from lidar elastic signal in $532 \mathrm{~nm}$ when the profile of particle backscatter coefficient had been known correctly. This method can also be used to get GFF for $1064 \mathrm{~nm}$ channel. As introduced above, the actual profile of particle backscatter coefficient in $1064 \mathrm{~nm}$ has to be known firstly. Note here, the color ratio of 532/1064 for aerosol backscatter coefficient above the height $\mathrm{z0}$ $(\mathrm{GFF}=1)$ can be used to obtain the "actual profile" in $1064 \mathrm{~nm}$ with the assumption of a fair homogenous atmosphere condition.

\section{RESULTS}

Measurement was made on Sep. 4, 2014 using our Mie lidar with side-scatter technique as shown in figure 2. In fig. 2(a), the aerosol profiles were obtained at 19:00 local time on September 04, 2014 when when the atmosphere condition is relative homogenous below the height of $1.0 \mathrm{~km}$ with an integrated AOD value 0.65 on the assumption of $\mathrm{S} 1$ equal 50 for $532 \mathrm{~nm}$. In the height of $0.8 \sim 1.5 \mathrm{~km}$, the aerosol backscatter coefficients from these two lidar techniques are almost the same. Below $0.8 \mathrm{~km}$, aerosol backscatter coefficients by Mie lidar are affected by the overlap factor. Through iteration algorithm, the inversed aerosol profiles by Mie lidar (blue line) and CCD side-scatter lidar (red line) are matched together perfectly. After that, the GFF for Mie lidar signal is obtained and shown in subfigure. In fig. 2(b), the aerosol profiles in 1064 nm are obtained comined Mie lidar and CCD sidescatter lidar through a homogenous atmosphere assumption (i.e., using the calculated color ratio and inversed backscatter coefficient for $532 \mathrm{~nm}$ by CCD lidar). Then the AOD value is 0.28 using $\mathrm{S} 1=40$ for $1064 \mathrm{~nm}$. The incomplete part affected by GFF is also given after iteration calculation as shown in sub-figure of Fig. 2(b). As to $1064 \mathrm{~nm}$ channel, the input parameters would induce uncertainty in determining GFF, receiver field of view and detector for two techniques are different, when there are particle layers on the laser paths, the measurements given by two techniques are probably different, which may be caused by the multi-scattering and different electronic response at the layer. Thus, the measurements should be performed under fair and homogenous atmosphere condition.
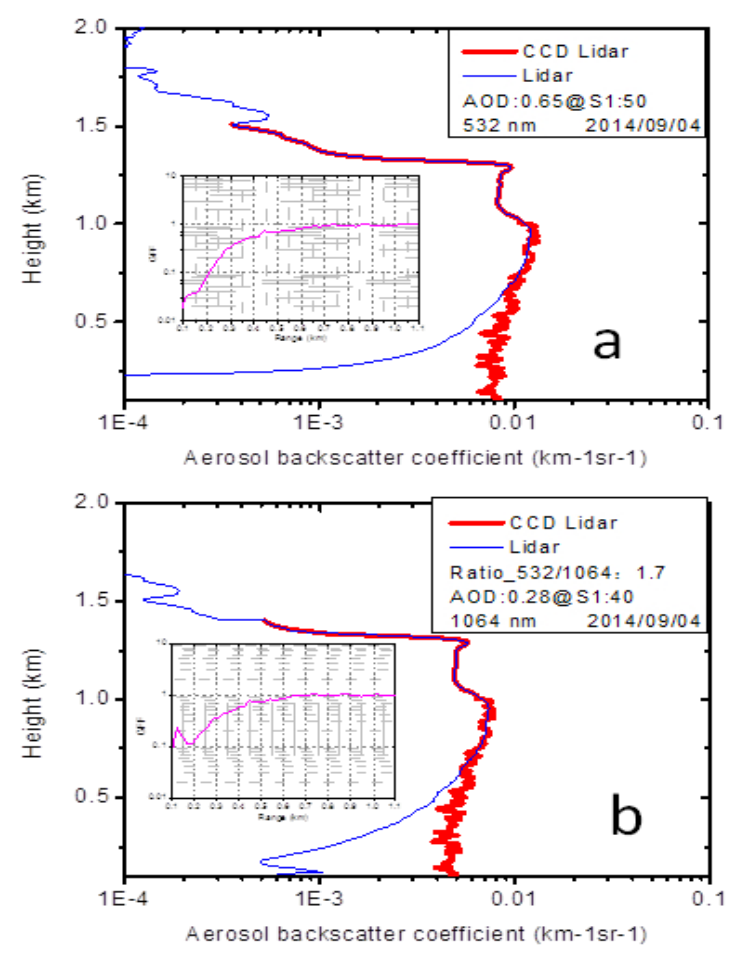

Figure 2. Particle backscatter coefficients obtained from CCD lidar (read line) and Mie lidar (blue line), and corresponding obtained GFF in sub-figure for 532 $\mathrm{nm}$ (a) and $1064 \mathrm{~nm}$ (b) channels on Sep. 04, 2014 over Hefei, China

\section{CONCLUSIONS}

In summary, a new experiment approach for determining overlap factor for vertically-pointing lidar is presented by iteration algorithm combining the retrieved particle backscatter coefficient using CCD side-scatter method and Fernald method. The effect of overlap factor on Mie lidar is corrected successfully in 532 and $1064 \mathrm{~nm}$ channels. This method is available in practice and GFF measurements for lidars can be determined easily in cloud-free night time by adding a CCD detector with a proper way. But for $1064 \mathrm{~nm}$, some assumption will be done, and multi-scattering may be another problem, further investigation will be carried out in the near future. 


\section{ACKNOWLEDGEMENT}

This research is supported by the National Natural Science Foundation of China (No. 41305022), the Strategic Priority Research Program of the Chinese Academy of Sciences (XDA05100302), and the Anhui Provincial Natural Science Foundation (No. 1308085MD53).

\section{REFERENCES}

[1]T. Halldórsson and J. Langerholc, "Geometrical form factors for the lidar function," Appl. Opt. 17, 240-244 (1978).

[2]K. Sassen and G.C. Dodd, "Lidar crossover function and misalignment effects," Appl. Opt. 21, 3162-3165 (1982).

[3]Y. Sasano, H. Shimizu, N. Takeuchi, and M. Okuda, "Geometrical form factor in the laser radar equation: an experimental determination," Appl. Opt. 18, 2886-2890 (1979).

[4]Ulla Wandinger and Albert Ansmann, "Experimental determination of the lidar overlap profile with Raman lidar," Appl. Opt. 41, 511-514 (2002).
[5]Shunxing Hu, Xiaobin Wang, Yonghua Wu, Chen $\mathrm{Li}$, and Huanling $\mathrm{Hu}$, "Geometrical form factor determination with Raman backscattering signals," Opt. Let. 30, 1879-1881 (2005).

[6] Z. Wang, Z. Tao, D. Liu, D. Wu, C. Xie, Y. Wang, A new experiment method for lidar overlap factor using a CCD side-scatter technique, Opt. Let. 40, (2015).

[7]J. E. Barnes, N. C. Parikh Sharma, and T. B. Kaplan, Atmospheric aerosol profiling with a bistatic imaging lidar system, Appl. Opt. 46(15), 2922-2929 (2007).

[8]F.G. Fernald, "Analysis of atmospheric lidar observations: some comments," Appl. Opt. 23, 652-653 (1984).

[9]Z. Tao, D. Liu, Z. Wang, X. Ma, Q. Zhang, C. Xie, S. Hu, Y. Wang, Measurements of aerosol phase function and vertical backscattering coefficient using a charge-couple device sidescatter lidar, Opt. Express 1127 DOI: 10.1364/OE.22.001127, (2014) 